\title{
Influência do Código de Pontuação no treino da Ginástica Artística Masculina ${ }^{1}$
}

\author{
Paulo Carrara ${ }^{1,2}$ \\ Luis Mochizuki $^{2,3}$ \\ ${ }^{1}$ Faculdade de Desporto, Universidade do Porto, Portugal \\ ${ }^{2}$ Escola de Educação Física e Esporte, Universidade de São Paulo, Brasil \\ ${ }^{3}$ Escola de Artes, Ciências e Humanidades, Universidade de São Paulo, Brasil
}

\begin{abstract}
Resumo: O objetivo deste estudo é verificar a influência das novas regras do Código de Pontuação na sistematização do treino da Ginástica Artística Masculina. Para compreender estas alterações, oito treinadores de seleções nacionais, de Portugal e Brasil, foram entrevistados, sobre como essas regras influenciam o treino. Foi utilizada a análise de conteúdo para avaliar os comentários. Os treinadores esclareceram que as novas regras implicaram em mudanças na estrutura das séries e manifestaram novas tendências na sistematização do treino.
\end{abstract}

Palavras-chave: Ginástica. Treino. Tendências.

\section{Influence of code of points in men's gymnastics training}

Abstract: The aim of this study is to verify the influence of Code of Points new rules on the systematization of Men's Artistic Gymnastics training. To understand these alterations, eight coaches of Portugal and Brazil national teams were questioned about how these rules affects the training. For the comments, a content analysis was run. The coaches mentioned that the new rules imply changes in routines composition and noticed new tendencies in training systematization.

Key Words: Gymnastics. Training. Trends.

\section{Introdução}

A Ginástica Artística Masculina (GAM) pressupõe tipos de movimentos diferenciados (ARAÚJO; MARQUES, 1995) e elevadas exigências para a execução (ARAÚJO, 2004), onde a técnica tem papel fundamental (FERREIRINHA, 2007; SMOLEVSKIY; GAVERDOVSKIY, 1996; UKRAN, 1978). As regras da GAM são determinadas pelo Código de Pontuação (CP) organizado pela Federação Internacional de Ginástica (FIG).

O CP define a filosofia geral da GAM, influencia e acompanha as mudanças para $O$ aperfeiçoamento da modalidade (FÉDÉRATION INTERNATIONAL DE GYMNASTIQUE, 2001; FÉDÉRATION INTERNATIONAL DE GYMNASTIQUE, 2006; NUNES, 2000).

Desde a criação do CP, em 1949, a nota máxima ou nota de partida (NP) foi 10 pontos (CARRARA, 2007). Em 2006, a FIG atualizou o $\mathrm{CP}$ (CP06) e promoveu mudanças significantes, oferecendo uma nova dimensão de critérios de avaliação na Ginástica Artística (GRANDI, 2005).

\footnotetext{
1 Trabalho derivado de Dissertação de Mestrado de Paulo Carrara na Faculdade de Desporto da Universidade do Porto - Portugal.
}

O CP06 acabou com o limite de 10 pontos na NP, e passou-se a atribuir pontos para os 10 elementos de maior dificuldade, mais 10 pontos pela apresentação de uma série completa (FÉDÉRATION INTERNATIONAL DE GYMNASTIQUE, 2006).

Historicamente, O CP passou a considerar a dificuldade dos elementos e séries como o critério de julgamento mais importante (MINUSA, 2000; NUNES, 2000). Entretanto, deve-se considerar outras características do CP06: a apreciação de no máximo quatro elementos do mesmo grupo estrutural na série, o aumento da dificuldade necessária para a saída e maior penalização para erros técnicos/posturais (FÉDÉRATION INTERNATIONAL DE GYMNASTIQUE, 2006). Especificamente aos aparelhos, é necessária a ligação de elementos de maior complexidade para que ocorra bonificação.

As mudanças no $\mathrm{CP}$ podem promover diferenças na estrutura das séries e modificar ou atualizar os métodos de treino (ARKAEV; SUCHILIN, 2004; GAJDOS, 1983; SMOLEVSKIY; GAVERDOVSKIY, 1996). Alguns autores (FERREIRINHA, 2007; MINUSA, 2000; READHEAD, 1997 ; SMOLEVSKIY; GAVERDOVSKIY, 1996) referem-se ao aumento 
da complexidade dos elementos e da dificuldade das séries apresentadas pelos ginastas no alto nível, e isto foi possível devido ao aumento no volume e intensidade do treino (ARKAEV; SUCHILIN, 2004; IRWIN et al., 2005; TRICOLI; SERRÃO, 2005).

O treino da GAM envolve a preparação física, técnica, tática e psicológica para que o ginasta assimile os elementos 3 e efetue as séries nos diferentes aparelhos. A preparação física fornece bases para a preparação técnica (UKRAN, 1978; WEINECK, 1999) e para a obtenção dos melhores resultados na Ginástica (GAJDOS, 1983, SMOLEVSKIY; GAVERDOVSKIY, 1996), como também atua na prevenção de lesões (ARAÚJO, 1998; ARAÚJO; MARQUES, 1995; TRICOLI; SERRÃO, 2005).

A preparação tática depende da preparação técnica, por ser utilizada no planejamento das séries (ARKAEV; SUCHILIN, 2004, GAJDOS, 1983), e busca a melhora dos resultados e a vitória. Os elementos da preparação tática devem ser abordados ao longo da preparação desportiva, e dependem das condições momentâneas do ginasta, sendo preferível a abordagem individual (GAJDOS, 1983).

A preparação psicológica deve estar combinada com a preparação física e técnica (READHEAD, 1997). No processo de treino, é essencial o desenvolvimento da força moral e a vontade necessária para suportar os treinos e evoluir no esporte (GRANDI, 2005; TRICOLI; SERRÃO, 2005), para garantir que a atividade realizada alcance um desempenho estável (ARKAEV; SUCHILIN, 2004).

Nesta perspectiva, espera-se que as séries sejam modificadas para atender as regras, e o treino adaptado para as modificações. Contudo, foram realizados poucos estudos para verificar tais fatos. Assim, o objetivo deste estudo é verificar quais as alterações foram realizadas nas séries e no treino após a atualização do CP06. Acredita-se que a opinião de especialistas pode auxiliar a definição e organização de métodos e conteúdos de treino, que levarão a melhoria do desempenho (TRICOLI; SERRÃO, 2005).

\section{Procedimentos Metodológicos}

\section{Características do estudo}

O presente estudo possui abordagem qualitativa, cujo objetivo foi identificar, em campo, características do treino da GAM de alto nível, sob influência de mudanças nas regras. No entanto, não foi intenção confrontar os dois grupos de técnicos, mas verificar aspectos do treino, específicos e generalizáveis, dentro de seus contextos.

\section{Participantes}

A escolha dos técnicos foi intencional, a fim de atender aos objetivos da pesquisa (PATTON, 2002). Foram delimitados oito treinadores de GAM de categoria adulto, dos quais quatro atuam em Portugal e quatro atuam no Brasil. A amostra representa os profissionais que no período de coleta de informações treinavam os melhores ginastas da categoria adulto em cada país. Trabalhavam com ginastas ou equipes que obtiveram numerosos resultados nacionais $\mathrm{e}$ internacionais, alguns deles recentes e os melhores alcançados até então. De referir que são treinadores responsáveis por ginastas pertencentes à equipe (seleção) que representa seu país em competições internacionais.

Tabela 1. Treinadores participantes.

\begin{tabular}{c|c|l}
\hline Treinador & $\begin{array}{c}\text { Tempo de } \\
\text { Atuação } \\
\text { (anos) }\end{array}$ & \multicolumn{1}{|c}{ Resultados como Treinador } \\
\hline TP1 & 30 & Qualificação para Jogos Olímpicos \\
\hline TP2 & 20 & Participação em Campeonato da Europa \\
\hline TP3 & 24 & Participação em Jogos Olímpicos \\
\hline TP4 & 18 & Participação em Jogos Olímpicos \\
\hline TB1 & 30 & Campeão Mundial por Aparelhos \\
\hline TB2 & 15 & Campeão Pan-americano por Aparelhos \\
\hline TB3 & 18 & Bicampeão Mundial por Aparelhos \\
\hline TB4 & 21 & Participação em Jogos Olímpicos \\
\hline
\end{tabular}

Os quatro treinadores em Portugal (TP) atuam na GAM no mínimo há 18 anos (média de 23,0 \pm 5,3 anos). Os quatro treinadores no Brasil (TB) atuam na GAM no mínimo há 15 anos (média de 21,0 \pm 6,5 anos) (Tabela 1). 


\section{Instrumento}

Foi utilizado um questionário de 12 itens, correspondentes às regras do $\mathrm{CP} 06$. A validade $\mathrm{e}$ fidedignidade foram verificadas por peritagem (MORROW et al., 2000) em Portugal e no Brasil, respectivamente pela avaliação e seleção do conjunto de regras mais importantes que representam o universo de regras contido no CP06. Foi verificada a concordância das respostas de três peritos em cada país, pela resposta de valor no mínimo três, em escala tipo Likert com cinco pontos (HILL; HILL, 2005), sendo 1 "nenhuma" até 5 "muitíssima" influência de cada item no treino da GAM (CARRARA, 2007). Para total compreensão dos treinadores, foram realizadas adaptações nos questionários, de modo a seguir a nomenclatura específica a cada país, de acordo com os respectivos CPs editados pelas federações nacionais.

\section{Procedimentos}

O questionário foi aplicado em entrevistas com a utilização de um gravador de voz Sony ICD-28, e para a transcrição software Digital Voice Editor 2. Todos os treinadores foram pessoalmente entrevistados por um dos autores deste trabalho (HILL; HILL, 2005).

Após estudo piloto com nove treinadores das categorias de base dos dois países, que comentaram sobre como cada item influiu nas séries e no treino, o questionário foi aplicado aos treinadores das seleções na categoria adulto, dando lugar a este trabalho de pesquisa.

Tabela 2. Itens do questionário em função do CP06.

\begin{tabular}{c|c|c}
\hline Banca D & Banca E & Aparelhos \\
\cline { 1 - 1 } Importância das Notas das Bancas D e E & \multirow{2}{*}{$\begin{array}{c}\text { Máximo de pontos } \\
\text { pela Banca E }\end{array}$} & $\begin{array}{c}\text { Ausência do bônus por ligação no Cavalo } \\
\text { com Arções e nas Paralelas }\end{array}$ \\
\cline { 1 - 1 } Elementos de dificuldade válidos & \multirow{2}{*}{$\begin{array}{c}\text { Valores das } \\
\text { penalizações }\end{array}$} & Bônus por ligação no Solo \\
\cline { 1 - 1 } Valor dos elementos & Bônus por ligação nas Argolas \\
\cline { 1 - 1 } Grupos de elementos & Bônus por ligação na Barra Fixa \\
\hline Saída D requerida &
\end{tabular}

Foram identificadas no questionário três partes específicas onde os itens estão agrupados de acordo com a classificação das regras do CP06: i) a Banca $D$, que é relativo às exigências na composição das séries; ii) a Banca $E$, que corresponde à avaliação da execução das séries; e iii) as regras específicas a cada Aparelho (Tabela 2).

\section{Análise de dados}

A partir da transcrição das entrevistas foi realizada uma análise descritiva, e com abordagem qualitativa, que pode funcionar sobre corpus de estudo reduzido e estabelecer categorias mais discriminadas (BARDIN, 2004). Essa análise não rejeita toda e qualquer forma de quantificação, o que a caracteriza é o fato da inferência ser fundada na presença do índice (tema, palavra), e não sobre a frequência de sua aparição, em cada comunicação individual.
O método selecionado para o tratamento dos dados da entrevista é a análise de conteúdo de Bardin (2004) que se realiza em três momentos. No primeiro ocorre a pré-análise, onde os dados são organizados, e ocorre a operação de recorte de texto em unidades de registro comparáveis, a categorização para a análise temática, e a codificação para o registro dos dados.

No segundo momento, ocorre a exploração do material: os dados são administrados em função das categorias propostas em cada questão ou grupo de questões. No terceiro momento, ocorre - tratamento e interpretação dos resultados obtidos. A análise temática é transversal, recorta o conjunto das entrevistas através de uma grelha de categorias projetada sobre os conteúdos. Os dados de conjunto dos discursos são considerados como segmentáveis e comparáveis (BARDIN, 2004). Assim, as respostas dos treinadores foram reunidas em uma estrutura de categorias e subcategorias (Tabela 3 ). 
Tabela 3. Estrutura de Categorização dos Conteúdos

\begin{tabular}{l|l|l}
\hline \multicolumn{1}{c|}{ Provas } & \multicolumn{1}{c|}{ Categorias } & \multicolumn{1}{c}{ Subcategorias } \\
\hline $\begin{array}{l}\text { - Solo } \\
\text { - Cavalo com Arções } \\
\text { - Argolas } \\
\text { - Paralelas } \\
\text { - Barra Fixa }\end{array}$ & Preparação Física & $\begin{array}{l}\text { - Força } \\
\text { - Resistência }\end{array}$ \\
\cline { 2 - 3 } & Preparação Técnica & $\begin{array}{l}\text { - Execução de elementos } \\
\text { - Grupos de elementos }\end{array}$ \\
\cline { 2 - 3 } & Preparação Tática & $\begin{array}{l}\text { - Complexidade da série } \\
\text { - Tamanho da série }\end{array}$ \\
\hline
\end{tabular}

\section{Resultados e Discussão}

Os temas emergentes referem-se às componentes do treino - Preparação Física, Técnica e Tática - e serão dispostos em função de suas subcategorias. Para todos os treinadores não houve qualquer mudança no treino exclusivamente para a prova de Salto sobre a Mesa.

Não houve um conjunto de dados suficiente para a projeção da categoria Preparação Psicológica, ou seja, para os treinadores não houve influência do CP06 nesta componente do treino. A maioria dos ginastas de elevado nível enfrentou várias situações adversas ao longo dos muitos anos da preparação competitiva específica, de modo que possuem práticos meios de autocontrole (ARKAEV; SUCHILIN, 2004).

\section{Preparação Física - Força}

Os treinadores de ambos os países deram mais importância em mudanças no trabalho de força para as argolas para atingir o bônus por ligação em elementos estáticos de força: "Tem que ser um trabalho diferente do $\mathrm{CP}$ antigo, a preparação física tem que ser muito especial" afirma TB2.

A força não foi citada pelos treinadores diante outras mudanças no CP06 porque se entende que essa capacidade é necessária para garantir o aprimoramento técnico (WEINECK, 1999). Sem um grau satisfatório de desenvolvimento de força não é possível aperfeiçoar os movimentos ginásticos, sendo requisito necessário que permite a preparação técnica dos elementos (ARAÚJO, 1998; ARAÚJO; MARQUES, 1995). Para os técnicos TP2, TP3 e TP4, alguns de seus ginastas não desenvolvem a capacidade força em níveis desejados, mesmo com o treino. Isto pode ocorrer devido a características genéticas (ARKAEV; SUCHILIN, 2004) da maioria dos ginastas de seu país. Assim, encontram contratempos maiores do que os esperados em desenvolver ligações em elementos de força nas argolas. O treino para este bônus está condicionado às capacidades adquiridas pelos ginastas ao longo de sua preparação plurianual (ARKAEV; SUCHILIN, 2004), e os exercícios de preparação específica devem ser escolhidos em função de suas particularidades individuais (UKRAN, 1978, WEINECK, 1999).

Por outro lado, TB1, TB2 e TB3 assumem que serão desenvolvidos novos elementos de força e ligações nas Argolas, o que poderá culminar com diferenças na composição das séries dos ginastas dos dois países.

\section{Preparação Física - Resistência}

O treino da resistência (anaeróbia) foi considerado mais influenciado pelo CP06 do que a força, na opinião dos treinadores de ambos os países. Para eles, as séries em todos os aparelhos ficaram maiores, e é necessário ter mais resistência para poder executar mais elementos de muita dificuldade e chegar a uma alta NP. Foi enaltecida a repetição de elementos e de séries, e a importância de conseguir realizar os elementos em combinação, pois "não adianta fazer um monte de elementos, para depois ter que juntá-los" afirma TB2.

Para os treinadores de ambos os países, o CP06 influenciou muito no treino da resistência, que deve ser maior, também para conseguir executar a saída de valor D (de grande dificuldade) no final da série (IRWIN et al., 2005), de modo a ter condições de apresentá-la adequadamente

(SMOLEVSKIY; GAVERDOVSKIY, 1996). "Se a dificuldade é maior, então influencia muito no treino" afirma TB1. "Para buscar o nível alto de qualidade das séries, o ginasta deve estar muito bem preparado fisicamente" afirma TB3, o que corrobora com a literatura (ARKAEV; SUCHILIN, 2004; 
FERREIRINHA, 2007; JEMNI et al., 2001). Cada aparelho demanda uma saída, e a das Argolas foi considerada por todos como a mais dificultada para se realizar ao final da série.

O Solo é o único aparelho com tempo máximo para apresentação, de até 70 segundos (FÉDÉRATION INTERNATIONAL DE GYMNASTIQUE, 2006). Mesmo com o tempo estipulado, todos os treinadores relataram que houve aumento do número de elementos e sequências acrobáticas nas competições. Isto tornou necessária a realização dos elementos com menor tempo de descanso. "O ponto fundamental é fazer a série com rapidez, pois é fácil passar do tempo máximo no solo, é importante a resistência" afirma TP1. É importante salientar que entre os aparelhos da GAM, o Solo é o de maior demanda fisiológica (JEMNI et al., 2001).

\section{Preparação Técnica - Execução de elementos}

Com a exposição inicial do CP06, parecia ser mais importante aos treinadores de ambos os países, a dificuldade. Entretanto "como pode haver muita dedução por incorreções, em vez de dar prioridade à dificuldade, tivemos atenção à execução" explica TP4. "A nota de apresentação valorizou a boa execução dos elementos" afirma TB4.

Os treinadores destacaram a execução dos elementos, e para isso planejam o treino sobre a parte técnica, que consideram fundamental, e não em função da dificuldade (UKRAN, 1978). "Eu me preocupo em fazer todos os elementos, em todos os aparelhos, praticando a execução perfeita. Se não consigo chegar lá, sempre volto atrás" diz TP3. A Preparação Técnica deve ser entendida como o trabalho da técnica do elemento através de aprendizagem e perfeccionismo (READHEAD, 1997; SMOLEVSKIY; GAVERDOVSKIY, 1996). Alguns fatores devem ser considerados a fim de propiciar uma melhor qualidade de treino, como, por exemplo, métodos adequados para 0 aperfeiçoamento das capacidades e das habilidades envolvidas no desenvolvimento dos elementos (TRICOLI; SERRÃO, 2005).

Para TP4 e TB4 o valor das penalizações do CP06 requer a execução perfeita dos elementos, para fazer provas com regularidade, o que é fundamental em termos competitivos. "Uma queda implica o atleta não ganhar" afirma TB1.
A maior importância da execução dos elementos ocorreu devido à mudança nos valores de penalização no CP06, que foram os maiores já aplicados (CARRARA, 2007). Isto é visto especialmente nas disciplinas como a GAM, onde a forma técnica é visualizada no desempenho (ARAÚJO; MARQUES, 1995; FERREIRINHA, 2007; IRWIN et al., 2005; WEINECK, 1999). Ao contrário de décadas atrás, atualmente os ginastas desenvolvem mais facilmente os elementos na sua máxima correção técnica possível, em função das suas características individuais e melhores condições de segurança (ARAÚJO, 2004).

\section{Preparação Técnica - Grupos de elementos}

Para todos os treinadores a regra que mais influenciou na estrutura da série, e portanto no treino, foi o limite máximo de quatro elementos do mesmo grupo estrutural. Isto influenciou muito, segundo TP1 e TB2, em treinar novos elementos e dificuldades de outros grupos, que o ginasta não treinava, para montar a série. Para eles o que ocorria era o ginasta fazer os tipos de elementos que tinha mais facilidade, e hoje é necessário fazer mais elementos de outros grupos para contar na NP: "Então obrigou a um leque muito diversificado na evolução técnica" afirma TP2. Foi necessário variar os elementos dos diferentes grupos, o que exige mais dos ginastas o domínio de um variado leque de técnicas gímnicas (ARAÚJO; MARQUES, 1995).

Quanto aos objetivos traçados sobre as novas regras no $\mathrm{CP}$, se houver ênfase de determinados elementos nas Provas, então será o que os treinadores vão procurar que seus ginastas façam (ARKAEV; SUCHILIN, 2004). A inclusão de elementos para compor a série depende das características dos ginastas, isto é, suas capacidades, morfologia ou qualidades técnicas (GAJDOS, $1983 ; \quad$ SMOLEVSKIY; GAVERDOVSKIY, 1996; READHEAD, 1997).

A Preparação Técnica do ginasta tem característica plurianual (ARKAEV; SUCHILIN, 2004, GAJDOS, 1983, SMOLEVSKIY; GAVERDOVSKIY, 1996), portanto as mudanças no treino necessitam de longos períodos para terem relevância, ou devem ser direcionadas aos ginastas desde as categorias de base. $O$ processo de aquisição de elementos variados $e$ de maior dificuldade é baseado no treino repetitivo e progressivo dos mesmos (TRICOLI; SERRÃO, 
2005; UKRAN, 1978), ocasionando o aumento do volume $e$ da intensidade de treino (FERREIRINHA, 2007, IRWIN et al., 2005).

\section{Preparação Tática - Complexidade da série}

$\mathrm{Na}$ opinião dos treinadores de ambos os países, a nota ilimitada da Banca $D$ foi uma das mudanças mais importantes no CP06, porque influiu na complexidade das séries e interferiu na Preparação Tática de seus ginastas.

Para eles, antes do CP06, a série era constituída de poucos elementos de alta dificuldade e algumas ligações para completar os 10 pontos máximos na NP, como também algumas coisas simples para cumprir as exigências. "Agora há números muito mais complexos" diz TP2, e os melhores ginastas poderão diferenciar-se (GRANDI, 2005) com uma série distinta e maior (IRWIN et al., 2005), conforme suas capacidades (GAJDOS, 1983). "A diferença na NP influencia totalmente no treino" diz TB1, corroborando com a literatura (ARKAEV; SUCHILIN, $\quad 2004 ; \quad$ SMOLEVSKIY; GAVERDOVSKIY, 1996). Muitos ginastas de alto nível executaram elementos cada vez mais difíceis e adicionaram a dificuldade ao extremo de suas capacidades (MINUSA, 2000), mas eram limitados pela nota máxima de 10 pontos.

Assim, segundo TP2 e TP3, inicialmente pensou-se que os resultados seriam definidos somente pela dificuldade representada na NP, e muita gente arriscou muitos elementos de alta dificuldade, mas falhou.

$O$ aumento dos descontos da Banca $E$ influiu também no modo como os treinadores compõem a série, que atualmente é determinado em função da relação entre a mais alta dificuldade que seu ginasta pode executar em detrimento da menor penalização. Assim, optaram por fazer uma apresentação com o melhor equilíbrio entre a dificuldade (Banca D) e apresentação (Banca E). Esta importância tática na composição da série se configura no treino da GAM pela busca do aumento da dificuldade, preservando a qualidade técnica (ARKAEV; SUCHILIN, 2004; READHEAD, 1997; SMOLEVSKIY; GAVERDOVSKIY, 1996).

A partir deste ciclo olímpico, ficaram evidenciados os objetivos que os treinadores de alto nível de ambos os países sempre buscaram: a perfeição da apresentação dos elementos, e não somente o valor de dificuldade dos mesmos
(GRANDI, 2005; UKRAN, 1978). Para os treinadores de Portugal, a partir das regras do CP06, muitos ginastas que têm uma boa média mundial fazem um bom nível de dificuldade e execução técnica, ou seja, expressam facilidade de execução (ARAÚJO, 2004). Isto mostra o equilíbrio entre a NP e a Nota Final, que é a filosofia da Ginástica (GRANDI, 2005).

Os treinadores de ambos os países destacaram que a dificuldade de uma série nunca deve ser forçada em detrimento de uma perfeita execução do ponto de vista técnico e postural (ARAÚJO, 2004), o que foi reforçado com o CP06 (GRANDI, 2005). Portanto, a composição das séries tem de corresponder às capacidades do ginasta, que deve ter domínio completo de seu corpo na apresentação de uma série em competição (FÉDÉRATION INTERNATIONAL DE GYMNASTIQUE, 2001). "Tem que explorar as capacidades dos ginastas, o máximo possível, mas com responsabilidade e bom senso" diz TP1. A definição de objetivos deve constituir preocupação dos treinadores, de forma a estabelecer e cumprir planos que se pretendem realistas em relação às características dos seus ginastas (ㅅAÚJO, 1998; READHEAD, 1997).

Para os treinadores TB1 e TP2, foi considerada também a inclusão de mais elementos de valores intermediários nas séries. Ao invés de fazer elementos de maior complexidade e ter penalizações, passaram a ser importantes os elementos de valor B e C.

No Cavalo com Arções e nas Paralelas as séries estão mais complexas, devido à retirada de bônus por ligação de elementos (FÉDÉRATION INTERNATIONAL DE GYMNASTIQUE, 2006). Muitos ginastas executavam os mesmos elementos e as mesmas ligações (ARKAEV; SUCHILIN, 2004), assim como séries similares, tornando os desempenhos cada vez mais semelhantes, o que poderia tornar a modalidade desinteressante (MINUSA, 2000).

Os treinadores de ambos os países alteraram a composição das séries nas Argolas, com a utilização de maior número de elementos de balanço, variando de acordo com o ginasta, para manter a NP elevada. A elaboração do programa de treino deve levar em consideração as necessidades específicas e as habilidades de cada ginasta (GAJDOS, 1983, TRICOLI; SERRÃO, 2005, UKRAN, 1978). Com o CP06 a série nas Argolas tem os elementos melhor 
distribuídos entre os grupos. É preciso fazer os elementos de força, mas também as partes de valor com balanço. "Argolas está muito menos aparelho de força agora" diz TB3. Para TP4 "Em norma geral, não temos ginastas com força para muitos elementos, então tentamos compensar com elementos de balanço, portanto não é assim tão prioritário, fazer elementos de força, quanto em CPs anteriores".

Na Barra Fixa não houve mudança no treino para realizar o bônus por ligação em elementos de largadas. Os ginastas podem treinar estes elementos, mas "depende muito da facilidade do ginasta em executar elementos de vôo, este tipo de elemento possui alto grau de risco e leva facilmente a erros nas séries" diz TB4. "Treinamos mais o que é mais valorizado, como também os elementos mais seguros" diz TP2. A composição da série e do treino deve ser organizada em função do aumento da dificuldade e da diminuição dos erros técnicos (ARAÚJO, 2004; SMOLEVSKIY; GAVERDOVSKIY, 1996), preservando a integridade do ginasta (FERREIRINHA, 2007).

Para os treinadores de ambos os países, há a possibilidade de uma série ter uma NP competitiva com elementos considerados mais seguros que as largadas, como os giros. Ou tenha elementos de vôo desde que o ginasta tenha competência em executá-los com regularidade. $\mathrm{O}$ princípio básico do $\mathrm{CP}$ é agora explícito, permite aos juízes identificar e atribuir valores aos ginastas mais talentosos e aos mais preparados (GRANDI, 2005).

\section{Preparação Tática - Tamanho da série}

Conforme todos os treinadores, a série ficou maior para se chegar a uma NP alta, principalmente no Cavalo com Arções e nas Paralelas. São executados muito mais elementos, e a série tem maior duração. Logo é necessário o cuidado para que a série não venha a conter um número excessivo de elementos, levando a um aumento das penalizações na apresentação, o que seria contrário ao colocado anteriormente pelos treinadores.

No Solo "é fundamental manter as sequências de elementos para conseguir bônus" diz TP4, pois há o limite, que é o tempo máximo de série (FÉDÉRATION INTERNATIONAL DE GYMNASTIQUE, 2006). Assim, "reduz a possibilidade do ginasta executar elementos puros de dificuldades superiores" diz TB4. Para TP3 "um ginasta pode facilmente buscar mais três ou quatro décimos por ligações, só por fazer os elementos em uma sequência... mas as ligações são para quem pode fazer, alguns ginastas não têm jeito". Assim, há maior ênfase nos bônus por ligações no Solo. Uma mudança considerável ocorre no treino quando são traçados novos objetivos (SMOLEVSKIY; GAVERDOVSKIY, 1996).

Com a importância aumentada da nota da Banca E na Nota Final, a inclusão de elementos na série está condicionada à execução com o mínimo de erros técnicos, pois os valores de penalização da Banca $E$ podem ser muito maiores que os valores de bônus (CARRARA, 2007; FÉDÉRATION INTERNATIONAL DE GYMNASTIQUE, 2006).

\section{Considerações Finais}

Com base nos resultados aqui apresentados, bem como nas interpretações desenvolvidas, concluiu-se haver tendências em ocorrer mudanças na estrutura das séries, proporcionadas pelas regras do CP06. As séries tiveram sua complexidade aumentada devido à limitação do número de elementos por grupo estrutural e ao maior número de elementos de grande dificuldade, inclusive a saída. Isto incidiu no aumento do volume e intensidade do treino, assim como em adaptações no conteúdo da Preparação Física, Técnica e Tática.

Em relação à Preparação Física, ocorreu principalmente $\mathrm{o}$ aumento do treino de resistência e força, em função das características das séries com mais elementos de dificuldade, e do desenvolvimento de novas combinações de elementos.

No que concerne à Preparação Técnica, verificou-se que o treino foi orientado para uma disposição técnica mais elaborada, com maior ênfase na qualidade de execução de diferentes tipos de elementos por grupo, em função dos maiores valores de penalização.

$\mathrm{Na}$ Preparação Tática, a complexidade da série foi valorizada pelos treinadores como estratégia principal para se manterem competitivos. Verificou-se que o CP06 influenciou a composição das séries, com elementos de dificuldade superior para obter o máximo de pontuação de dificuldade, bem como numa 
execução ótima dos elementos para alcançar a nota máxima de execução.

Os treinadores buscam que seus ginastas incluam o máximo de elementos de dificuldade em suas séries. Entretanto, este conceito é delimitado pelo valor aumentado das penalizações e pelo limite de elementos por grupo, como também o fato de que os treinadores apreciam realizar um trabalho que preserve a integridade dos ginastas.

Assim, frente ao ocorrido com CPs anteriores, a partir do CP06 os treinadores entrevistados preocupam-se mais com a organização das séries, de modo a manter o equilíbrio entre a dificuldade e a execução dos elementos para aumentar a NP.

$\mathrm{Na}$ adaptação das séries às regras do CP06 foram verificadas diferenças entre as metodologias de treino utilizadas pelos treinadores de alto nível dos dois países, mais evidentemente nas Argolas. Portanto, é importante salientar que as variações existentes no planejamento do treino dependem de outros fatores além do $\mathrm{CP}$, como as características fisiológicas dos ginastas e suas respostas individualizadas ao treino.

Estudos adicionais, relacionados à sistematização do treino de ginastas em categorias competitivas de base, poderiam fornecer outras evidências e hipóteses mais claras para verificar se houve influência do CP06 na formação dos mais jovens em função da perfeita execução técnica, assim como a aprendizagem de diferentes tipos de elementos em todos os Aparelhos.

\section{Referências}

ARAÚJO, C. O treino dos jovens ginastas. Horizonte, Lisboa, v. 15, n. 85, p.1-12, 1998.

ARAÚJO, C. Manual de ajudas em Ginástica. Porto: Porto Editora, 2004.

ARAÚJO, C.; MARQUES, A. Modelo de participação competitiva em Ginástica Desportiva Masculina. Horizonte, Lisboa, v. 12, n. 68 p.5561, 1995.

ARKAEV, L.; SUCHILIN, N. Gymnastics: How to Create Champions. Oxford: Meyer \& Meyer Sport, 2004.

BARDIN, L. Análise de conteúdo. Lisboa: Edições 70, 2004.
CARRARA, P. Influência do Código de Pontuação na sistematização do treino da Ginástica Artística Masculina: estudo com treinadores de alto nível de Portugal e Brasil. 2007. 167 f. Dissertação (Mestrado em Educação Física) - Faculdade de Desporto, Universidade do Porto, Porto, 2007.

FÉDÉRATION INTERNATIONAL DE GYMNASTIQUE. Code of points: Artistic gymnastics for men. Suisse: FIG, 2001.

FÉDÉRATION INTERNATIONAL DE GYMNASTIQUE. Code of points: Artistic gymnastics for men. Suisse: FIG, 2006.

FERREIRINHA, J. O Modelo de Carga Externa em GAF de Alto Rendimento - A estrutura e as tendências evolutivas dos exercícios de competição em Paralelas Assimétricas. 2007. $294 \mathrm{f}$. Tese (Doutorado) - Departamento de Desporto, Universidade de Trás-os-Montes e Alto Douro, Vila Real, 2007.

GAJDOS, A. Préparation et entraînement à la gymnastique sportive. Paris: Éditions Amphora, 1983.

GRANDI, B. Letter from the president. Suisse: Fédération International de Gymnastique, 2005.

HILL, M.; HILL, A. Investigação por questionário. Lisboa: Edições Sílabo, 2005.

IRWIN, G.; HANTON, S.; KERWIN, D. The conceptual process of skill progression development in artistic gymnastics. Journal Sports Sciences, Abingdon, v. 23, n. 10, p.10891099, 2005.

JEMNI, M.; FRIEMEL, F.; SANDS, W.; MIKESKY, A. Evolution du profil physiologique des gymnastes durant les 40 dernieres annees. (revue de littérature). Canadian Journal Applied

Physiology. Ottawa, v. 26, n. 4, p.356-370, 2001.

MINUSA, H. How We Must Develop the Sport of Gymnastics for the Future. Technique, Indianápolis, v. 20, n. 8, p.16-18, 2000.

MORROW, J.; JACKSON, A.; DISCH, J.; MOOD, $D$. Measurement and evaluation in human performance. Champaign, IL: Human Kinetics, 2000.

NUNES, M. Reavivando as Memórias, 50 anos. Lisboa: Federação Portuguesa de Ginástica, 2000.

PATTON, M. Qualitative research \& evaluation methods. Thousand Oaks: Sage, 2002. 
READHEAD, L. Men's gymnastics coaching manual. Marlborough, UK: The Crowood Press, 1997.

SMOLEVSKIY, V.; GAVERDOVSKIY, I. Tratado general de gimnasia artística deportiva.

Barcelona: Paidotribo, 1996.

TRICOLI, V.; SERRÃO, J. Aspectos Científicos do Treinamento Esportivo Aplicados à Ginástica Artística. In: NUNOMURA, M.; NISTA-PICCOLO, V. (Ed.). Compreendendo a ginástica artística. São Paulo: Phorte, 2005. p.143-152

UKRAN, M. Metodología de entrenamiento de los gimnastas. Zaragoza: Editorial Acribia, 1978.

WEINECK, J. Treinamento Ideal. São Paulo: Editora Manole, 1999.

Apoio: Convênio internacional FADE-UP e EEFEUSP.

Agradecimentos: Hilton, Cristiano, Blanco, Ricardo, Robson, Tiago, Cláudio, Washington, Marcos, Renato, Leonardo, Pastor, Ksaky, Nunes, Pedro, Álvaro, Ferreirinha, Alda, Eunice, Araújo, Botelho e Guilherme (em memória).

Endereço:

Paulo Carrara

Escola de Educação Física e Esporte - USP

Av. Prof. Mello Moraes, 65 - Butantã

São Paulo SP Brasil

05508-900

Telefone: (11) 9796-7069

e-mail: paulocarrara@usp.br

Recebido em: 29 de março de 2010.

Aceito em: 8 de setembro de 2011.

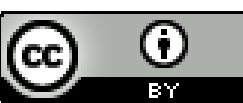

Motriz. Revista de Educação Física. UNESP, Rio Claro, SP, Brasil - elSSN: 1980-6574 - está licenciada sob Creative Commons - Atribuicão 3.0 